\title{
The effects of bowl size and portion size on food intake and fullness ratings in a sample of Japanese men
}

\author{
Misa Shimpo ${ }^{1, *}$ and Rie Akamatsu ${ }^{2}$ \\ ${ }^{1}$ Faculty of Health and Human Development, Department of Food and Health Sciences, The University of Nagano, \\ 8-49-7 Miwa, Nagano City, Nagano 380-8525, Japan: ${ }^{2}$ Faculty of Core Research, Natural Science Division, \\ Ochanomizu University, Tokyo, Japan
}

Submitted 5 March 2018: Final revision received 13 June 2018: Accepted 18 June 2018: First published online 06 August 2018

\begin{abstract}
Objective: Portion sizes and bowl sizes may be related to food intake and perceived fullness. The objective of the present study was to investigate the effects of portion size and bowl size and possible interactions between these variables on food intake and fullness in a sample of Japanese men.

Design: Participants ate four different experimental meals across four weeks and completed questionnaires about their fullness using a visual analogue scale administered before and after meals. The four meal patterns included consistent portions of several foods commonly eaten together in typical Japanese meals, along with $150 \mathrm{~g}$ of rice served in a small rice bowl (diameter of $11.5 \mathrm{~cm}$ ), $150 \mathrm{~g}$ of rice served in a large rice bowl (diameter of $13.5 \mathrm{~cm}$ ), $250 \mathrm{~g}$ of rice served in a small rice bowl or $250 \mathrm{~g}$ of rice served in a large rice bowl.

Setting: Tokyo.

Subject: Twenty-one adult men participated in the study.

Results: Portion size had a significant main effect on rice intake $\left(F_{(1,20)}=83\right.$, $P<0 \cdot 001)$ and fullness $\left(F_{(1,20)}=8 \cdot 0, P=0 \cdot 010\right)$, but no significant effects of bowl size on the outcome variables were found. The interactions between portion size and bowl size on intake and fullness were not significant.

Conclusions: The sample of Japanese men showed an influence of portion size on food intake. Further research is needed to clarify the combined effects of bowl size and portion size on intake and fullness.
\end{abstract}

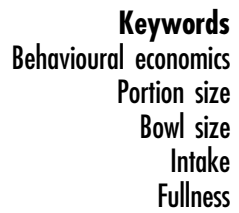

Strategies for controlling food consumption are important for weight management and the prevention of chronic health conditions like obesity and type 2 diabetes. Recently, behavioural economics has been identified as a source of strategies to help control food consumption. Behavioural economics applies theories from cognitive psychology to understand how people make decisions; according to a systematic review of behavioural interventions to promote intake of healthy foods, achieving healthy food intake at recommended levels will require stronger behavioural interventions that draw knowledge from other approaches, such as the theory of behavioural economics $^{(1,2)}$. In fact, Song et al. ${ }^{(3)}$ found that a nutrition programme that combined a behavioural economics approach and nutrition education led to increased consumption of healthy foods.

According to behavioural economics, decisions about which foods to eat, and how much to eat, rely on fast and automatic cognitive processes ${ }^{(4)}$. Previous studies have found that decisions about what and how much to eat are linked to the order or placement of food on shelves in stores $^{(5,6)}$, labelling of food items ${ }^{(7)}$, economic incentives such as discount sales ${ }^{(7)}$ and portion size ${ }^{(8)}$. These strategies have been called 'nudges', implying that they are a way to push slightly or gently to get someone's attention or prod them into action; several such strategies have a proven influence on diet and food consumption.

In terms of controlling the amount of food consumption, portion size is important. A previous study reported that intakes of total fat, saturated fat, cholesterol and sodium of customers were lower when both reduced-size and fullsize entrées were offered as alternatives than when only full-size entrées were offered in a worksite cafeteria and a commercial upscale restaurant ${ }^{(8)}$. Meta-analyses covering many countries clarify the effects of portion size on food consumption $^{(9,10)}$. Although the systematic review included several studies, the only study conducted in Asia was in South Korea. Regarding differences in portion size by country, Rozin et al. ${ }^{(11)}$ suggested that portion sizes in the USA were larger than in France. With respect to package 
size, Poelman et $a l .{ }^{(12)}$ suggested that substantial withinand between-country variation exists in recommended serving sizes for sweet drinks across Australia, Canada, the Netherlands and New Zealand.

Japanese people, in particular, have a characteristic food culture and manners during meals. For example, almost all Japanese eat rice every day as a staple food, and they learn at home and at school that leaving food on the plate is bad manners ${ }^{(13)}$. On the other hand, leaving food indicates gratitude for hospitality in China; there, it means that one ate until completely full. There is a distinct possibility that the effect of portion size on food consumption among Japanese differs from that among people in other countries in Asia and elsewhere.

The effects of previous interventions manipulating portion sizes have been only small to moderate, leading to calls for more effective interventions, according to reviews ${ }^{(14,15)}$. To develop effective interventions involving reducing portion size, researchers have studied numerous factors and mechanisms to understand the relationship between portion size and food consumption ${ }^{(15-17)}$. 'Appropriateness' is one such mechanism, which states that people interpret the portion they are served as being of an appropriate size and eat the entire portion accordingly. Another is 'visual cues', which posits that when more food is present, more will be consumed. Next, 'previous experience/expectation' states that a person's previous experience of eating a food, and the degree of fullness previously obtained, influences the portion size selected and consumed at later times. Finally, 'external factors' also play a role in food consumption; these factors include the value of food for the money paid, levels of awareness and estimation bias.

Recently, there have been many studies about plate size associated with visual cues. When a circle is enclosed in a larger circle that is proximal it will be perceived as larger and when distal, smaller; this phenomenon is called the Delboeuf illusion $^{(18)}$. The Delboeuf illusion influences eating behaviours because most people use a plate at their meals, and people tend to feel a large meal in a small plate and a small meal in a large plate. Other research has demonstrated that plate size can affect satiation and expectations of satiation. Specifically, one study ${ }^{(19)}$ found that a small plate was associated with higher estimated satiation and lower estimated intake than a larger plate and suggested that this was because the portions appeared different to the eater. Other studies have also investigated the effects of plate size on food intake and fullness, but their results have been inconsistent ${ }^{(20-25)}$. According to a systematic review, the difference in food consumption between small and large plate conditions was marginally statistically significant, but effect magnitude was small because of inconsistent results ${ }^{(26)}$; subgroup analysis suggested that participant characteristics (weight status), type of dish (plate or bowl), place of experiment (outside or inside a laboratory) and food type (snack or main meal) may influence these results. In other studies, sex and nationality influenced the relationship between dish size and food consumption, but there are few studies on this in Asian countries, including Japan ${ }^{(27)}$.

Many studies have investigated behavioural economic factors on food consumption and food choice. Recently, the effects of combinations of more than two behavioural economic factors have come to be investigated. Wilson et al. ${ }^{(6)}$ found that packaging and placement order influenced food choice. The combination of behavioural economic factors may be more effective on food consumption than single effects and have an interactive effect on food consumption.

The purpose of the present study was to address gaps in the extant literature regarding behavioural economic factors, eating behaviours and other cultural factors that can influence food consumption. More specifically, the goal was to address the respective and interactive effects of portion size and bowl size on intake and fullness among Japanese men. In Japan, the rate of obesity among men is higher than it is for women ${ }^{(28)}$ and white rice contributes most to total daily energy intake in the Japanese diet ${ }^{(28)}$. Therefore, the study targeted Japanese men and focused on rice and rice bowls.

\section{Methods}

\section{Participants}

The participants were workers at Ochanomizu University recruited through advertising leaflets and email announcements sent by faculty members at the university. Due to the nature of the study, to avoid demand characteristics, participants were not told the study objective; instead they were told that they would be taking part in a survey about flavour preferences. The true objective of the study was not told through the surveys to avoid demand characteristics. Eligible participants were men between the ages of 20 and 60 years, without any food allergies to the meals provided in the study. On the advertising leaflets and email, it was stated that the study contained four times experimental meals and questionnaires before and after meals to examine their flavour preferences. Before the experiments, the participants were informed that they could withdraw from the study at any time.

\section{Experimental design}

Participants ate lunch in a classroom at the university and answered questionnaires before and after the meals once per week for a period of four weeks (Fig. 1). Participants were asked to not to change their typical lifestyle habits (eating behaviours and physical activity) on the day before each study day. Experimental meals were scheduled at the same time of day each time. Participants were asked to eat 


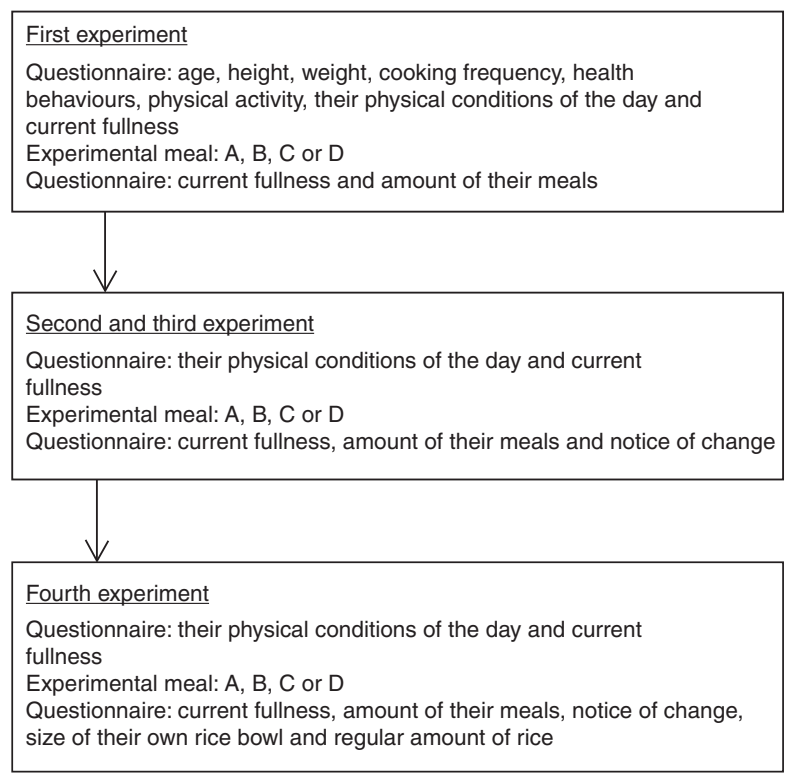

Fig. 1 Study procedure. Participants ate four different experimental meals and answered questionnaires before and after the meals, once per week, for four weeks

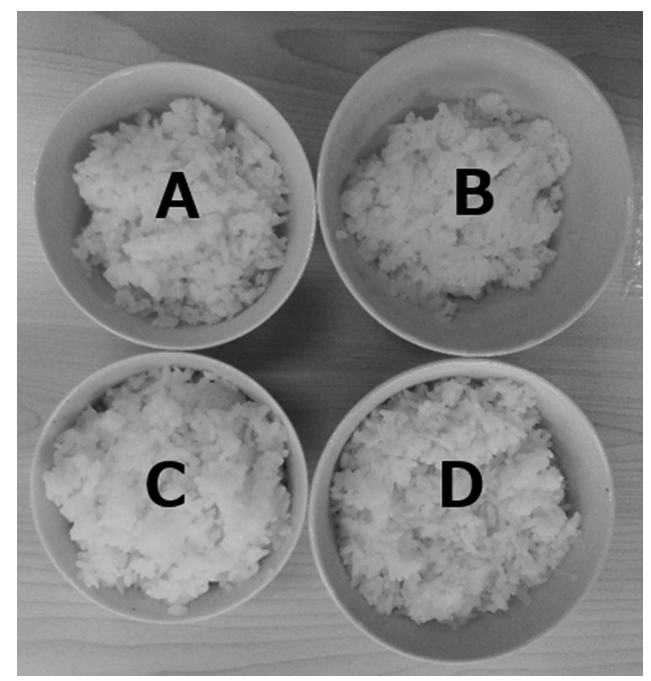

Fig. 2 The two sizes of rice bowl and two amounts of rice used in the present study. Rice bowls $A$ and $C$ were $11.5 \mathrm{~cm}$ in diameter. Rice bowls $B$ and $D$ were $13.5 \mathrm{~cm}$ in diameter. The amount of rice served in $A$ and $B$ was $150 \mathrm{~g}$. The amount of rice served in $C$ and $D$ was $250 \mathrm{~g}$

the same breakfast on each study day and not to consume any foods and beverages in the hour before lunch.

Each week, participants were guided to a specified table in the room and given one of the four possible meals, named A, B, C and D (Figs 2 and 3). The meal labels were not seen by participants, to disguise the purpose of the study. Participants were seated at separate tables, facing in the same direction, so that they could concentrate on their meals. Meal A contained $150 \mathrm{~g}$ of rice in a small rice bowl (diameter of $11.5 \mathrm{~cm}$ ). Meal B contained $150 \mathrm{~g}$ of rice in a large rice bowl (diameter of $13.5 \mathrm{~cm}$ ). Meal C contained

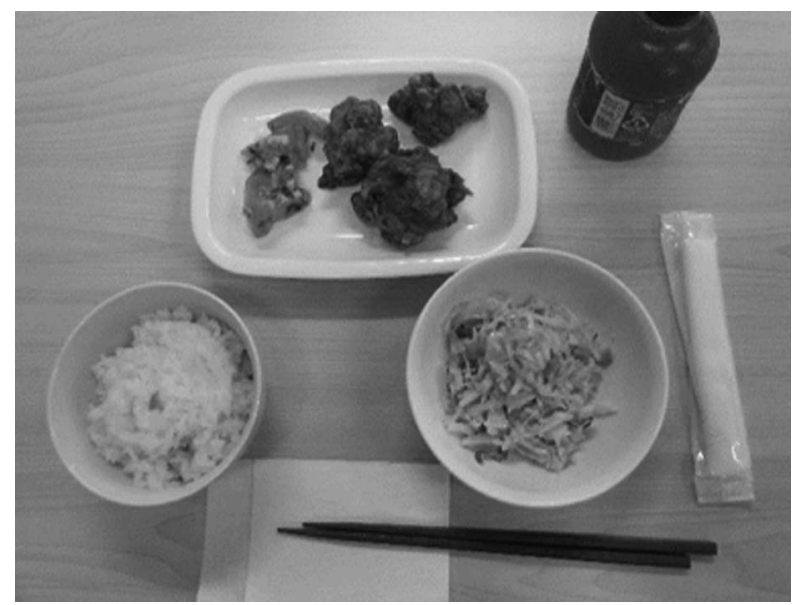

Fig. 3 Experimental meal (Meal A). The experimental meal included rice, deep-fried chicken, tomato, coleslaw and green tea. The size of the rice bowl and the amount of rice changed over the four experimental meals

$250 \mathrm{~g}$ of rice in a small rice bowl. Meal D contained $250 \mathrm{~g}$ of rice in a large rice bowl. The order in which participants were given the different meals (rice bowl sizes and amounts of rice) was randomly assigned to adjust for order effects, using random numbers in Microsoft ${ }^{\circledR}$ Excel. Participants were not explicitly informed of the changes in bowl size or portion size; they were later asked if they noticed any differences. In addition to the rice, participants were served the same foods and beverage in the same amounts: $120 \mathrm{~g}$ of deep-fried chicken ( $1473 \mathrm{~kJ}$ ( $352 \mathrm{kcal})$ ), $50 \mathrm{~g}$ of tomato salad $(92 \mathrm{~kJ}(22 \mathrm{kcal})), 100 \mathrm{~g}$ of coleslaw $(297 \mathrm{~kJ}(71 \mathrm{kcal}))$ and $280 \mathrm{ml}$ of green tea $(0 \mathrm{~kJ}(0 \mathrm{kcal}))$. The total energy of the dishes except the rice was $1862 \mathrm{~kJ}$ $(445 \mathrm{kcal})$. The amounts of rice and other foods were in accordance with the 'Japanese Health Meal' developed by the national Ministry of Health, Labour and Welfare ${ }^{(29)}$. Meals A and B were $2916 \mathrm{~kJ}$ (697 kcal) each; Meals C and D were $3619 \mathrm{~kJ}$ (865 kcal) each. Participants were instructed to eat as much of the meal as they wanted and were then permitted to obtain refills on as much rice as they wanted by raising their hand. The amount of rice participants took when getting refills was weighed. Because Japanese people tend to hesitate when it comes to refilling their bowls or leaving the table immediately after eating, the researchers encouraged the participants to not feel embarrassed and to take more rice at any time, and to feel free to leave the table when they felt that the amount of rice taken was more than they could eat. After the participants finished eating and left the classroom, the foods left over were weighed using a digital cooking scale (Tanita KD-320-WH).

\section{Measures}

Before and after each experimental meal, participants answered questionnaires. The questionnaire before the first experimental meal included items on age, height, 
weight, cooking frequency, health behaviours (four items) and physical activity. Health behaviour items covered the following topics: whether or not the participant ate to the point of fullness, refrained from eating fatty foods, refrained from eating high-carbohydrate foods and ate a lot of vegetables. The four responses were 'always', 'sometimes', 'rarely' and 'never'. Furthermore, to disguise the objective of the study, participants were asked to report their flavour preferences. Each questionnaire that was administered before meals included the participant's physical conditions of the day. They also rated their fullness using a 100-point visual analogue scale.

Questionnaires were administered after participants consumed the meals. They rated their fullness using the 100-point visual analogue scale. They were asked at each meal 'How much was the amount of your meal you were served?' and answered from five responses which were 'very little', 'little', 'appropriate', 'a lot' and 'a whole lot'. To disguise the purpose of the study, participants also rated their flavour preference for the meal using the question 'How did it taste?' The five responses were 'most favourite flavour', 'favourite flavour', 'between favourite and unfavourite', 'unfavourite' and 'most unfavourite'. After the second time the participants visited the room to eat, they were asked whether they noticed differences between the first and the second experimental session, and were provided with free descriptive space to write their responses. On the fourth survey (only), after participants had consumed the meal and completed the questions (which were the same questions asked after Meals 2 and 3), they were shown a large and small rice bowl and asked to rate the size of their own bowl using the following responses: 'smaller than the smaller rice bowl', 'the same size as the smaller rice bowl', 'between the smaller rice bowl and the larger one', 'the same size as the larger rice bowl' and 'larger than the larger rice bowl'. The average amount of rice in the participant's rice bowl was assessed by having participants choose one of five illustrations that most closely matched their standard eating habits (respectively showing $110 \mathrm{~g}, 140 \mathrm{~g}, 165 \mathrm{~g}, 200 \mathrm{~g}$ and $230 \mathrm{~g}$ in the same size rice bowl) used by the Japanese National Health and Nutrition Survey ${ }^{(30)}$.

\section{Statistical analysis}

Sample size was calculated using G*Power 3.1.9.2 software $^{(31)}$ for two-way repeated-measures ANOVA between and within factors and for within-between interactions. A power analysis calculation indicated that with two factors and two levels, for power of 0.8 and effect size $(f)$ of 0.25 , described as medium by Cohen $^{(32)}$, at least seventeen participants were required to conduct experimental meals four times each.

Fullness was calculated by subtracting fullness before the meal from fullness after the meal. Repeated-measures ANOVA was used to investigate effects and interactions between portion size and bowl size on food intake and fullness. All analyses were conducted using the statistical software package IBM SPSS Statistics for Windows, version 19.0. Statistical significance was set at $P<0 \cdot 05$.

\section{Results}

Complete study data were obtained from twenty-one participants. The characteristics of participants are shown in Table 1. Five participants (24\%) were overweight based on their BMI (BMI $\geq 25 \cdot 0 \mathrm{~kg} / \mathrm{m}^{2}$ ). As noted above, participants were allowed to refill only with rice; it was refilled five times over the total of eighty-four meals. The number of times rice remained was two; other dishes were never left unfinished. The mean fullness before the meal across four meals was 26.0 (SD 12.8) and after the meal, 86.1 (SD 6.2). The mean fullness before and after for Meal A (small rice bowl, small amount of rice served) was 27.1 (SD 17.0) and 84.3 (sD 6.8), respectively. For Meal B (large rice bowl, small amount of rice served), it was 28.8 (SD 17.1) and 84.3 (SD 7.5), respectively; for Meal C (small rice bowl, large amount of rice served), $20 \cdot 7$ (SD 13.4) and 88.3 (SD 7.5), respectively; and for Meal D (large rice bowl, large amount of rice served), 27.4 (sD 19.9) and 87.6 (sD 8.9), respectively.

Responses on whether amounts were appropriate are shown in Table 2. About $80 \%$ of participants responded that the amounts of tomato, coleslaw and chicken were

Table 1 Characteristics of the participating Japanese men ( $n$ 21), September to October 2017

\begin{tabular}{|c|c|c|}
\hline & Mean or $n$ & SD or $\%$ \\
\hline Age (years), mean and SD & 39.9 & $9 \cdot 8$ \\
\hline $\mathrm{BMI}\left(\mathrm{kg} / \mathrm{m}^{2}\right)$, mean and SD & 23.6 & 2.4 \\
\hline \multicolumn{3}{|l|}{ Physical activity, $n$ and $\%$} \\
\hline Low & 6 & 29 \\
\hline Moderate & 14 & 67 \\
\hline High & 1 & 5 \\
\hline \multicolumn{3}{|l|}{ Cooking frequency, $n$ and $\%$} \\
\hline Never & 6 & 29 \\
\hline One or two days per week & 6 & 29 \\
\hline Three to five days per week & 7 & 33 \\
\hline Six or more days per week & 2 & 10 \\
\hline \multicolumn{3}{|l|}{ Health behaviours* ${ }^{*} n$ and $\%$} \\
\hline Stopping eating before I get full & 15 & 71 \\
\hline Refraining from fatty foods & 11 & 52 \\
\hline Refraining from high-carbohydrate foods & 9 & 43 \\
\hline Eating a lot of vegetables & 16 & 76 \\
\hline \multicolumn{3}{|l|}{ Own rice bowl size, $n$ and $\%$} \\
\hline Smaller than the smaller rice bowl & 2 & 10 \\
\hline The same size as the smaller rice bowl & 10 & 48 \\
\hline Between smaller rice bowl and larger one & 5 & 24 \\
\hline The same size as the larger rice bowl & 2 & 10 \\
\hline Larger than the larger rice bowl & 2 & 10 \\
\hline \multicolumn{3}{|l|}{ Regular amount of rice, $n$ and \% } \\
\hline $110 \mathrm{~g}$ & 6 & 29 \\
\hline $140 \mathrm{~g}$ & 5 & 24 \\
\hline $165 \mathrm{~g}$ & 4 & 19 \\
\hline $200 \mathrm{~g}$ & 5 & 24 \\
\hline $230 \mathrm{~g}$ & 1 & 5 \\
\hline
\end{tabular}

‘The number and rate of participants who answered 'always' or 'sometimes'. 
Table 2 Perception of the amount of each dish among the sample of Japanese men ( $n$ 21), September to October 2017

\begin{tabular}{|c|c|c|c|c|c|c|c|c|c|c|}
\hline & \multicolumn{2}{|c|}{ Very little } & \multicolumn{2}{|c|}{ Little } & \multicolumn{2}{|c|}{ Appropriate } & \multicolumn{2}{|c|}{ A lot } & \multicolumn{2}{|c|}{ A whole lot } \\
\hline & $n$ & $\%$ & $n$ & $\%$ & $n$ & $\%$ & $n$ & $\%$ & $n$ & $\%$ \\
\hline \multicolumn{11}{|l|}{ Rice } \\
\hline$A(150 \mathrm{~g})$ & 1 & $4 \cdot 8$ & 3 & $14 \cdot 3$ & 16 & $76 \cdot 2$ & 1 & 4.8 & 0 & 0.0 \\
\hline$B(150 \mathrm{~g})$ & 1 & 4.8 & 6 & $28 \cdot 6$ & 13 & 61.9 & 1 & $4 \cdot 8$ & 0 & 0.0 \\
\hline$C(250 \mathrm{~g})$ & 0 & 0.0 & 0 & 0.0 & 11 & $52 \cdot 4$ & 9 & $42 \cdot 9$ & 1 & $4 \cdot 8$ \\
\hline $\mathrm{D}(250 \mathrm{~g})$ & 0 & 0.0 & 1 & 4.8 & 9 & $42 \cdot 9$ & 9 & $42 \cdot 9$ & 2 & 9.5 \\
\hline Tomato salad & 1 & $1 \cdot 2$ & 9 & $10 \cdot 7$ & 73 & 86.9 & 1 & $1 \cdot 2$ & 0 & 0.0 \\
\hline Coleslaw & 1 & $1 \cdot 2$ & 6 & $7 \cdot 1$ & 66 & $78 \cdot 6$ & 11 & $13 \cdot 1$ & 0 & 0.0 \\
\hline Deep-fried chicken & 1 & $1 \cdot 2$ & 11 & $13 \cdot 1$ & 67 & $79 \cdot 8$ & 5 & $6 \cdot 0$ & 0 & 0.0 \\
\hline
\end{tabular}

Rice $A$ to $D$, number of observations $=21$; tomato salad, coleslaw and deep-fried chicken, number of observations $=84$.

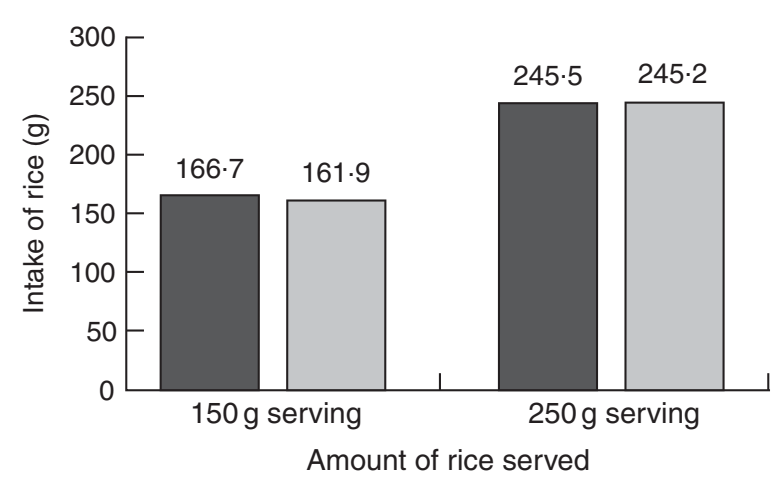

Fig. 4 Intake of rice from the four experimental meals by the sample of Japanese men ( $n$ 21), September to October 2017. The amount of rice served had a significant main effect on intake of rice in the meal $(P<0.001)$. The main effect of bowl size ( $\square$, small; $\square$, large) and the interaction of the amount of rice served and bowl size on intake were not significant

appropriate, while about half the participants responded that the amount of rice ( $250 \mathrm{~g}$ in Meals $\mathrm{C}$ and D) was 'a whole lot' or 'a lot'.

Overall, fourteen of the participants (67\%) reported that they noticed changes in the amount of rice that they were given across the four study sessions. For fifteen of the participants, the amount of rice given in the meals decreased from the first to the last study session. Twelve of these participants $(80 \%)$ reported that they noticed this decrease in the amount of rice. For sixteen of the participants, in contrast, the amount of rice increased from the first to the last meal; nine of these participants (56\%) reported that they noticed an increase in the amount of rice. None of the participants reported noticing a change in the size of their rice bowl.

The amount of rice served had a significant main effect on intake of rice during the meal $\left(F_{(1,20)}=83, P<0 \cdot 001\right.$; Fig. 4), whereas bowl size did not $\left(F_{(1,20)}=0.36\right.$, $P=0.555$ ). The mean intake of rice was 166.7 (SD 45.0 ) $\mathrm{g}$ when $150 \mathrm{~g}$ of rice was served in a small rice bowl, 161.9 (SD 37.6) g when $150 \mathrm{~g}$ was served in a large rice bowl, 245.5 (SD 20.7) g when $250 \mathrm{~g}$ was served in a small rice bowl and 245.2 (SD 22.0) g when $250 \mathrm{~g}$ was served in a large rice bowl. There was no significant interaction between the amount of rice served and the size of the rice bowl on the intake of rice $\left(F_{(1,20)}=0 \cdot 28, P=0 \cdot 601\right)$. As for mean energy intake from the meals (overall), that with the $150 \mathrm{~g}$ serving in the small bowl amounted to $3033 \mathrm{~kJ}$ $(725 \mathrm{kcal})$, that with $150 \mathrm{~g}$ in a large rice bowl was $3000 \mathrm{~kJ}$ $(717 \mathrm{kcal})$, that with $250 \mathrm{~g}$ in a small rice bowl was $3586 \mathrm{~kJ}$ $(857 \mathrm{kcal})$ and that with $250 \mathrm{~g}$ in a large rice bowl was $3586 \mathrm{~kJ}(857 \mathrm{kcal})$.

The amount of rice served had a significant main effect on fullness $\left(F_{(1,20)}=8 \cdot 0, P=0 \cdot 010\right.$; Fig. 5), whereas bowl size did not $\left(F_{(1,20)}=2 \cdot 2, P=0 \cdot 155\right)$. Mean fullness was $57 \cdot 2$ (SD 17.2) when $150 \mathrm{~g}$ of rice was served in a small bowl, $55 \cdot 5$ (SD 16.8) with $150 \mathrm{~g}$ of rice in a large bowl, 67.6 (SD 16.2) with $250 \mathrm{~g}$ in a small bowl and 60.2 (SD 17.7) with $250 \mathrm{~g}$ in a large bowl. The interaction between the amount of rice served and the size of the rice bowl on rice intake was not statistically significant $\left(F_{(1,20)}=1 \cdot 7\right.$, $P=0 \cdot 207)$.

\section{Discussion}

The results of the present study suggest that the amount of rice served affected rice intake and self-rated fullness among Japanese men. However, neither the main effects of bowl size on intake and fullness nor those of interactions between amount of rice served and bowl size on intake and fullness were significant. More than half of participants noticed a change in the amount of rice during the study, but no participants noticed the change in the size of the rice bowl.

The intake and fullness of participants increased when they were served larger portion sizes; this result is consistent with previous studies ${ }^{(8-10,14,15)}$ and suggests that individuals are likely to overeat if they are served more food. More than half of participants answered that their regular amount of rice was less than $150 \mathrm{~g}$, but only a few people left their rice when they were served $250 \mathrm{~g}$ rice. There are cultural considerations to take into account here, since it is considered poor table manners in Japan to leave 


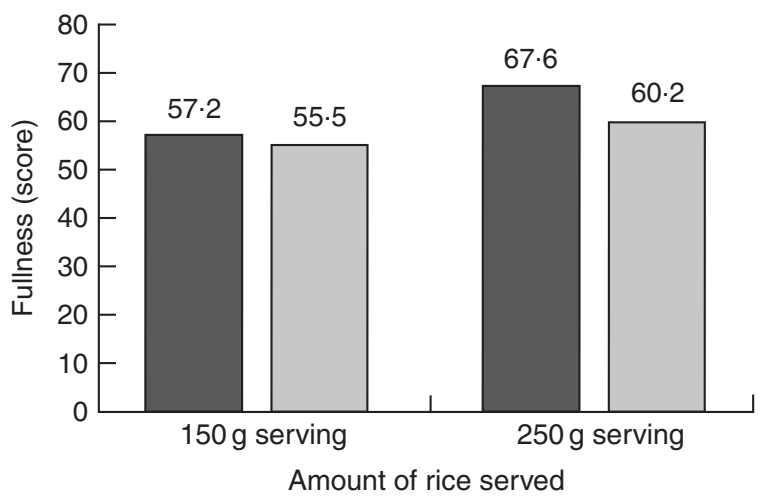

Fig. 5 Fullness rating of the four experimental meals by the sample of Japanese men ( $n$ 21), September to October 2017. The amount of rice served had a significant main effect on fullness $(P<0.001)$. The main effect of bowl size $(\square$, small; $\square$, large) and the interaction of the amount of rice served and bowl size on fullness were not significant.

part of a meal unfinished ${ }^{(13)}$. Tradition has it that there are seven gods in a grain of rice in Japan, so many Japanese people feel guilty leaving rice. In addition, the Japanese value mottainai, an ancient Buddhist term from Japan that means respect for surrounding resources, not wasting resources and using them with a sense of gratitude. The current study may help understand the significance of the amount of food served and its ability to control one's meals, worldwide and particularly in Japan. While the study adds to the literature, however, additional research is needed to better understand the effects of portion size on intake and fullness among Japanese individuals.

Previous studies have reported no consistent effects of bowl size on food intake ${ }^{(20-26)}$ and our results align with this previous research, while suggesting that the effect of portion size was larger than the effect of bowl size in Japanese men. In Japan, people sometimes share meals from large plates and each eats using a small plate. Previous studies suggest that serving behaviour has a direct influence on plate size $\mathrm{i}^{(18,33)}$. The interactive effects of portion size and plate size on serving behaviour also need research.

The interactions between portion size and bowl size on intake and fullness were not significant. Across the experimental meals, fullness was highest when the larger portion size was provided in the smaller rice bowl and lowest when the smaller portion size was provided in the larger rice bowl. This interaction may be borne out in future studies with larger samples sizes and/or larger differences in portion size and bowl size. In this regard, it is possible that the present study was susceptible to type II error due to the small sample size. However, this can nevertheless be considered an appropriate pilot investigation of this phenomenon; particularly the fact that participants did not notice the change in bowl size should be helpful for future researchers.
The present study has several additional limitations. First, participants ate their meals in the classroom, which was different from the contexts in which most people would generally eat their meals. In a real-world setting, many people socialize while eating and this may divert their attention from the food and portion sizes. As such, future research would do well to investigate these behaviours in more natural settings. Another limitation is that the study focused only on men and only on consumption of one specific food (rice); there may be effects of different dishes. Future research investigating these patterns in Japanese women and/or with other types of food is needed. Third, another limitation is in the size(s) of the portions and dishes used. In the present study, only a few participants refilled their bowl or left any rice. The smaller amount of rice served may not be small enough to elicit refill, while the large amount may be not large enough to need to leave some. Thus, future study should use less than $150 \mathrm{~g}$ and more than $250 \mathrm{~g}$ rice. In a related point, previous studies have used larger dishes $^{(22-24,27)}$; it is possible that larger difference in bowl size may lead to greater effect of bowl size on intake amount.

\section{Conclusions}

The present study suggests that the portion size of rice had effects on intake and fullness ratings among Japanese men; it was the first study of the effects of portion size and bowl size on intake and fullness among Japanese men. Although the main effects of bowl size and the interactions between portion size and bowl size were not clear in the study, they may perhaps be seen with larger sample sizes and/or different populations and foods. Further research should clarify the effects of portion size, bowl size and their interaction to better understand strategies for controlling food consumption.

\section{Acknowledgements}

Financial support: This study was supported by JSPS KAKENHI (grant number JP 17H00713). JSPS KAKENHI is a public research fund and had no role in the design, analysis or writing of this article. Conflict of interest: None. Authorship: M.S. designed the study, analysed the data and wrote the initial draft of the manuscript. R.A. assisted in designing the study and critically reviewed the manuscript. Both authors contributed to data collection and interpretation, and approved the final version of the manuscript and any revised version. Ethics of buman subject participation: This study was conducted according to the guidelines laid down in the Declaration of Helsinki and all procedures involving human subjects were approved by the Ethics Committee of Ochanomizu 
University (accepted number: 2017-9). Written informed consent was obtained from all subjects.

\section{References}

1. Bickel WK, Moody L \& Higgins ST (2016) Some current dimensions of the behavioral economics of health-related behavior change. Prev Med $\mathbf{9 2}, 16-23$.

2. Thomson CA \& Ravia J (2011) A systematic review of behavioral interventions to promote intake of fruit and vegetables. J Am Diet Assoc 111, 1523-1535.

3. Song HJ, Grutzmacher S \& Munger AL (2016) Project ReFresh: testing the efficacy of a school-based classroom and cafeteria intervention in elementary school children. $J$ Sch Health 86, 543-551.

4. Kahneman D (2011) Thinking, Fast and Slow. New York: Farrar, Straus and Giroux.

5. Hanks AS, Just DR, Smith LE et al. (2012) Healthy convenience: nudging students toward healthier choices in the lunchroom. J Public Health 34, 370-376.

6. Wilson NL, Just DR, Swigert J et al. (2017) Food pantry selection solutions: a randomized controlled trial in clientchoice food pantries to nudge clients to targeted foods. J Public Health 39, 366-372.

7. Olstad DL, Goonewardene LA, McCargar LJ et al. (2014) Choosing healthier foods in recreational sports settings: a mixed methods investigation of the impact of nudging and an economic incentive. Int J Behav Nutr Phys Act 22, 11,6 .

8. Berkowitz S, Marquart L, Mykerezi E et al. (2016) Reduced-portion entrées in a worksite and restaurant setting: impact on food consumption and waste. Public Health Nutr 19, 3048-3054.

9. Zlatevska N, Dubelaar C \& Holden SS (2014) Sizing up the effect of portion size on consumption: a metaanalytic review. J Mark 78, 140-154.

10. Hollands GJ, Shemilt I, Marteau TM et al. (2015) Portion, package or tableware size for changing selection and consumption of food, alcohol and tobacco. Cochrane Database Syst Rev issue 9, CD011045.

11. Rozin P, Kabnick K, Pete E et al. (2003) The ecology of eating: smaller portion sizes in France than in the United States help explain the French paradox. Psychol Sci 14, 450-454.

12. Poelman MP, Eyles H, Dunford E et al. (2016) Package size and manufacturer-recommended serving size of sweet beverages: a cross-sectional study across four high-income countries. Public Health Nutr 19, 1008-1016.

13. Ministry of Education, Culture, Sports, Science and Technology (2010) Guidance of Nutrition Education. http:// www.mext.go.jp/a_menu/sports/syokuiku/1292952.htm (accessed February 2018).

14. Wright B \& Bragge P (2018) Interventions to promote healthy eating choices when dining out: a systematic review of reviews. Br J Health Psychol 23, 278-295.

15. Steenhuis I \& Poelman M (2017) Portion size: latest developments and interventions. Curr Obes Rep 6, 10-17.

16. English L, Lasschuijt M \& Keller KL (2015) Mechanisms of the portion size effect: what is known and where do we go from here? Appetite 88, 39-40.
17. Peter HC, Herman C, Polivy J et al. (2015) Mechanisms underlying the portion-size effect. Physiol Behav 144, 129-136.

18. Van Ittersum K \& Wansink B (2012) Plate size and color suggestibility: the Delboeuf illusion's bias on serving and eating behavior. J Consum Res 39, 215-228.

19. Peng M (2017) How does plate size affect estimated satiation and intake for individuals in normal-weight and overweight groups? Obes Sci Pract 27, 282-288.

20. Ayaz A, Akyol A, Cetin C et al. (2016) Effect of plate size on meal energy intake in normal weight women. Nutr Res Pract 10, 524-529.

21. Libotte E, Siegrist M \& Bucher T (2014) The influence of plate size on meal composition. Literature review and experiment. Appetite 82, 91-96.

22. Robinson E, Sheen F, Harrold J et al. (2016) Dishware size and snack food intake in a between-subjects laboratory experiment. Public Health Nutr 19, 633-637.

23. Rolls BJ, Roe LS, Halverson KH et al. (2007) Using a smaller plate did not reduce energy intake at meals. Appetite $\mathbf{4 9}$, 652-660.

24. Shah M, Schroeder R, Winn W et al. (2011) A pilot study to investigate the effect of plate size on meal energy intake in normal weight and overweight/obese women. J Hum Nutr Diet 24, 612-615.

25. Stroebele-Benschop N, Dieze A \& Hilzendegen C (2016) First come, first served: does pouring sequence matter for consumption? Appetite 105, 731-736.

26. Robinson E, Nolan S, Tudur-Smith C et al. (2014) Will smaller plates lead to smaller waists? A systematic review and meta-analysis of the effect that experimental manipulation of dishware size has on energy consumption. Obes Rev 15, 812-821.

27. Peng M, Adam S, Hautus MJ et al. (2017) See food diet? Cultural differences in estimating fullness and intake as a function of plate size. Appetite 117, 197-202.

28. Ministry of Health, Labour and Welfare (2017) The National Health and Nutrition Survey in Japan, 2015. http://www. mhlw.go.jp/bunya/kenkou/eiyou/dl/h27-houkoku-01.pdf (accessed February 2018).

29. Ministry of Health, Labour and Welfare (2015) Healthy Meals; Guide of Meals for Preventing Non-communicable Disease and Promoting Health. http://www.mhlw.go.jp/ file/04-Houdouhappyou-10904750-Kenkoukyoku-Gantaisa kukenkouzoushinka/0000096859.pdf (accessed February 2018).

30. National Institute of Health and Nutrition (2009) Book of Food Portion Sizes 2009. http://www.nibiohn.go.jp/eiken/ chosa/pdf/scale2009_2013ver.pdf\# search=\%27\%E6\%A0\% 84\%E9\%A4\%8A\%E6\%91\%82\%E5\%8F\%96\%E7\%8A\%B6\% Е6\%B3\%81\%Е8\%AA\%BF\%E6\%9F\%BB\%Е3\%81\%AE\%Е3\% 81\%9F\%E3\%82\%81\%E3\%81\%AE\%27 (accessed February 2018).

31. Faul F, Erdfelder E, Buchner A et al. (2009) Statistical power analyses using G*Power 3.1: tests for correlation and regression analyses. Behav Res Methods 41, 1149-1160.

32. Cohen JA (1992) A power primer. Psychol Bull 112, 155-159.

33. Wansink B, van Ittersum K \& Painter JE (2006) Ice cream illusions bowls, spoons, and self-served portion size. Am J Prev Med 31, 240-243. 